\title{
STUDY ON A NEW KIND OF ELECTROMAGNETIC MECHANICAL COUPLED REGENERATIVE BRAKING SYSTEM FOR ELECTRIC VEHICLES
}

\author{
Jincui LIU \\ Xinxiang Vocational And Technical College, Xinxiang City, Henan province, China \\ email:_incuiliujcl@163.com
}

\begin{abstract}
Electric vehicles technology is an effective way to solve the automobile energy and pollution problems, and regenerative braking is one of the advantageous technologies to achieve electric vehicles high braking energy recovery and to improve the energy efficiency. On the basis of electromagnetic theory and braking energy flow analysis, combined with modern advanced electromechanical control technology, we put forward a new kind of electromagnetic mechanical coupled braking system (EMCB), and make simulation experiments for longitudinal stability control of the system. The results showed that: the proposed ABS continuous state control strategy based on the new EMCB system improved the shortcomings of the traditional ABS discrete switch control, and avoided the high frequency chattering vibration of the system, with good response, robustness and slip rate control performance, which improves the braking energy recovery and braking comfort and effectively increases the driving range of electric vehicles.
\end{abstract}

Keywords: electric vehicle; electromagnetic mechanical coupled braking; ABS continuous state control.

\section{Introduction}

With the rapid increase of the world car ownership, in the face of the pressure of clean energy and the ecological environment, the automobile industry needs to solve energy, security, pollution, congestion and so on problems. Countries around the world began to develop new energy vehicles, continuously introduce relevant policies to promote new energy vehicles, especially the technology breakthroughs and industrial development of electric vehicles. Europe and other vehicles developed countries have put forward the electric power, low-carbon, and intelligent development goal, and implemented the integration of technological innovation, crossindustry collaboration and so on to accelerate and promote the automobile industry to achieve transformation and change under the background of developing a new generation of clean energy technology and information Internet technology.

Electric vehicle technology is an effective way to solve the energy and pollution problems of the automobiles [1]. Regenerative braking is easy to achieve efficient braking energy recovery, which is an important way to improve the utilization rate of electric vehicle energy $[2,3]$.
Especially in the frequent start and stop of the urban condition, the braking deceleration is mainly distributed in the range of $0 \sim 0.159 \mathrm{~g}$ [4], and the use of regenerative braking can improve the mileage for $14 \% \sim 40 \%$ [5].

According to the statistics, when the car is driving in urban conditions, the braking process is about $50 \%$ of the driving energy loss, and in the suburbs conditions, there are about $20 \%$ of the energy loss [6]. As a result, to improve the efficiency of braking energy recovery is beneficial to reduce energy consumption, decrease friction loss, and extend the driving range and so on, which has an important significance in increasing mileage of electric vehicles. The vehicle braking system mainly includes air braking, hydraulic braking, electromagnetic braking and so on.

The electromagnetic braking used on vehicles includes electromechanical braking system (EMB), motor regenerative braking system, eddy current retarder and so on. Regenerative braking can not only recover the braking energy and improve energy efficiency, but also has rapid response and high control precision compared with the hydraulic and pneumatic mechanical braking methods. The braking torque is easy to realize fast and accurate control, and can decrease friction and reduce the effects of thermal recession on braking performance in the vehicle braking process. 


\section{Methods}

\subsection{Braking mechanism of electromagnetic friction}

Electromagnetic theory is an important theoretical support to promote the development of modern science and technology, especially in the field of communication and industry. In the communication field, electromagnetic field theory is taken as the basis; in the industrial field, electromagnetic effect is taken as the basis. In the industrial field, motor, eddy current retarder, eddy current dynamometer and so on, through the electromagnetic effect, realize the conversion of mechanical energy and electric energy, electromagnetic brake, magnetic powder clutch and magnetorheological and so on, by electromagnetic effect, achieve the conversion of mechanical energy and thermal energy.

According to the electromagnetic theory and the regenerative braking and friction braking energy flow analysis, we integrate modern advanced electromechanical control technology, and combined with the mechanism of electromechanical braking and regenerative braking of the motor, put forward a new kind of electromagnetic-mechanical coupled regenerative braking system, hereinafter referred to as EMCB. The system integrates the advantages of EMB, wheel motor regenerative braking and direct distribution drive, and overcomes the disadvantages of two sets of brake system mutually independent and not same response characteristics. In the electromagnetic brake energy feedback, it motivates friction brake and realizes electromagnetic mechanical coupled regenerative braking, whose energy flow is shown in Figure 1 . The electromagnetic force on the rotor acts on the wheel and generates the electromagnetic braking force, so the vehicle kinetic energy is transformed into electrical energy; at the same time, the electromagnetic force, by hydraulic transmission or spiral transmission and so on institutions, generates actuation force to motivate the friction brake, and constitutes a coupled brake with electromagnetic brake, so the vehicle kinetic energy is transformed into thermal energy.

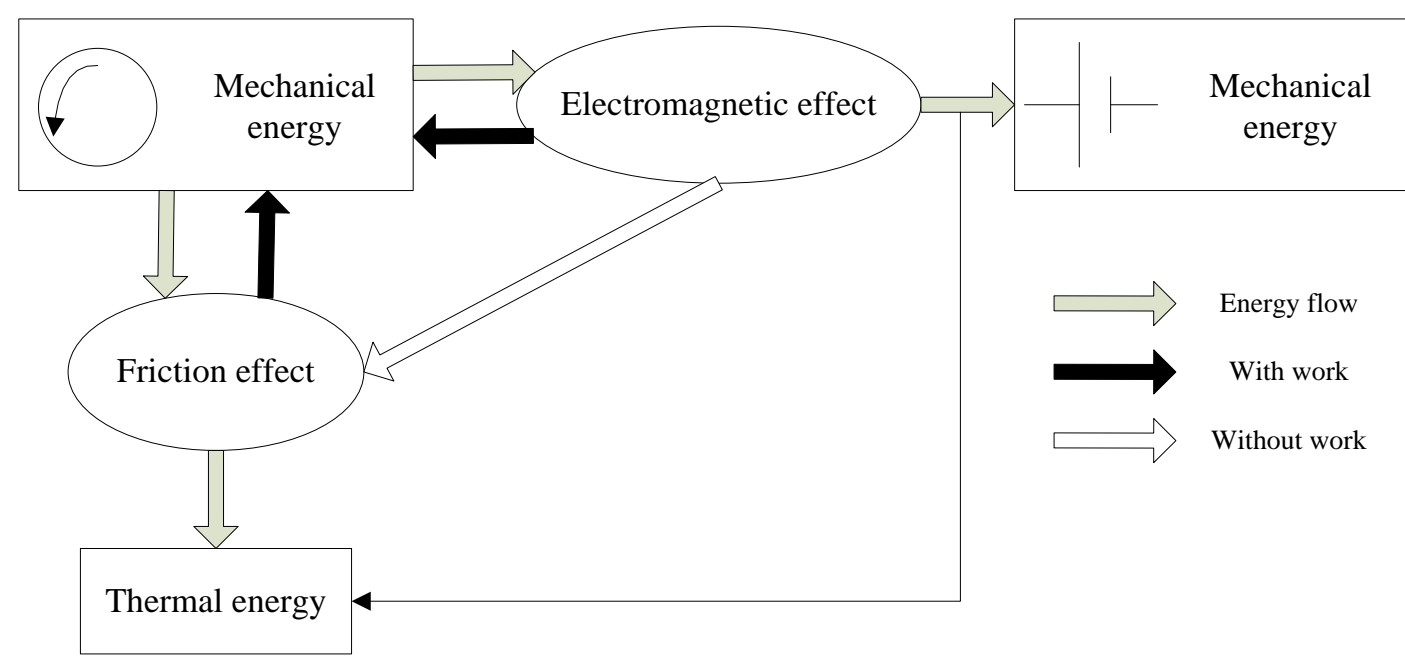

Figure 1: Electromagnetic mechanical coupled regenerative braking system energy flow

When braking, the motor is in the state of electricity generation, and the motor control drives the motor controller to generate electricity for charging the electricity storage device. The electromagnetic torque between the rotor and the stator of the motor is transmitted to the wheels through a transmission device, which hinders the rotation of the wheels and makes the wheels and the ground producing braking force; according to Newton's Third Law, the electromagnetic torque between the motor rotor and the stator is also acted on the motor stator, which is passed to the screw through a spline shaft sleeve consolidated with a motor stator. The clutch device is in off state, and the spline sleeve is supported on the fixing device and it can rotate freely. The screw cooperates with the spline sleeve through the spline sliding and the screw cooperates with the nut by non-self-locking, and the guide block limits rotation of the nut so that the nut only conducts axial slip. As a result, the screw driven by spline sleeve and the nut reversely slide along the axial direction. The right end thrust bearing is restricted by the fixed device for the axial displacement, and the nut or the screw can only promote the axial movement of the left thrust bearing. When the electromagnetic torque on the motor stator, through the driving force generated by the screw and the nut mechanism, can overcome the elastic force of the elastic element, and eliminate the gap between the thrust bearing and the brake caliper, it will push the brake disc to produce friction torque and transfer to the wheels from the motor 
rotor through error clamping and transfer the wheel of the motor rotor through the pin and transmission device consolidated on the motor rotor, which hinders the rotation of the wheels, and makes the wheel and the ground producing force; the electromagnetic torque of motor rotor and the friction torque of the brake disc form the coupling brake torque of the braking wheels.

When driving, the motor is in the state of power consumption, and the motor controller controls the storage device to provide power for the motor. The electromagnetic torque between the rotor and the stator of the motor, through a transmission device, is transmitted from the motor rotor to the wheels, driving wheels to turn and make the wheels and the ground to produce the driving force; the clutch device is in the state of combination, and the spline sleeve is supported on the fixing device and fixed through the clutch device. As a result, the electromagnetic torque acted on the motor stator is not transmitted to the screw nut mechanism, it does not produce spiral thrust, and the friction brake system does not work; the drive shaft is supported on the fixing device and it can rotate freely. The engine or the central driving motor power is transmitted from the drive shaft to the wheels through a transmission device, to drive the wheel to rotate and make the wheel and the ground producing driving force; the electromagnetic torque of the motor rotor and the driving torque of the transmission shaft form the composite driving torque for the driving wheels.

\subsection{Braking force equation}

Interactive acting force between the rotor and the stator of the motor, through the brake wheels of a transmission device, overcomes the elastic torque of preset elastic elements, and then drives the screw driving device through a spline shaft sleeve. We can obtain the following relationship:

$\left\{\begin{array}{l}\omega_{m}=i_{c 1} \omega_{L g} \\ \omega_{m}=i_{c 2} \omega_{w} \\ T_{1}=i_{c 1}\left(T_{L}-T_{s}\right) \\ T_{b e}=i_{c 2} T_{L}\end{array}\right.$

In (1), $\omega_{m}$ and $\omega_{w}$ are the motor mechanical angular velocity and the wheels angular velocity of motor, respectively, $\mathrm{rad} / \mathrm{s} ; i_{c 1}$ and $i_{c 2}$ are the transmission ratio from the motor to the screw spline and the transmission ratio from the motor to the wheels, respectively; $T_{1}, T_{L}, T_{b e}$, and $T_{s}$ are the screw drive torque, motor brake torque, magnetic brake torque, and the elastic moment of preset elastic elements $\mathrm{N} \cdot \mathrm{m}$.
The braking torque of the motor overcomes the elastic torque to drive the screw transmission device to produce the spiral thrust force, and the relation between the screw thrust and the motor braking torque and the elastic moment can be obtained by (1) and the screw transmission equation (2):

$$
F_{N}=\frac{T_{1}}{R_{c 5} \cdot \tan \left(\alpha_{s}+\rho_{s}\right)}
$$

In (2), $T_{1}$ represents the screw drive torque $\mathrm{N} \cdot \mathrm{m} ;{ }^{F_{N}}$ refers to the screw or nut driving force, N.

$$
F_{N}=\frac{\eta_{1} i_{c 1}\left(T_{L}-T_{s}\right)}{R_{c 5} \cdot \tan \left(\alpha_{s}+\rho_{s}\right)} \cdot \operatorname{sgn}\left(T_{L}-T_{s}\right)
$$

In (3), $F_{N}$ suggests the screw thrust, N; $\eta_{1}$ indicates the mechanical efficiency of the screw drive device.

When the screw thrust is not enough to overcome the elastic force of the rear elastic elements to eliminate the brake clearance, the spiral thrust and spring elastic are mutual acting force and reaction force, and friction brake is not involved in; when the spiral thrust reaches the peak point $F_{s 0}$ of the rear elastic element, the brake clearance is completely eliminated. With the continuous increase of the screw thrust, the thrust and the spring elastic force are no longer the mutual acting force and reaction force, and the friction braking starts to act. In consequence, the relationship between the brake pressure and the screw thrust and the elastic force can be expressed as:

$$
F_{c}=\left\{\begin{array}{cc}
0 & \left(F_{N} \leq F_{s 0}\right) \\
F_{N}-F_{s} & \left(F_{N}>F_{s 0}\right)
\end{array}\right.
$$

In (4), $F_{c}$ indicates the pressure acted on the brake disc, N; $F_{s 0}$ 为 refers to the elastic force N of the corresponding rear elastic elements when the brake clearance is completely eliminated; $F_{s}$ represents the diaphragm elastic force, $\mathrm{N}$; other parameters are similar to those above.

Motor brake torque $T_{L}$, through the front elastic element and the screw driving device, produces the brake pressure $F_{c}$ acted on the friction wheel to motivate the friction brake. The friction braking torque $T_{b c}$ and the electromagnetic braking torque $T_{b e}$ acted on the wheels, as shown in Figure 2, constituting a total braking torque of EMCB system. 


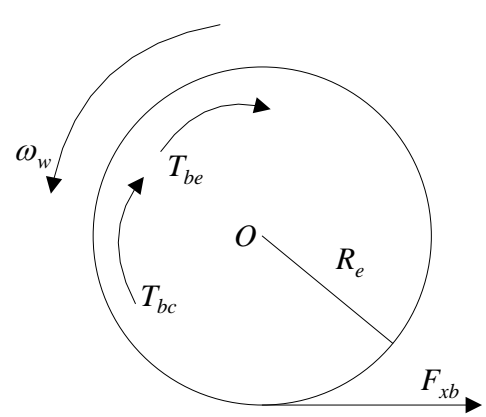

Figure 2: Wheel braking process force diagram

The relationship among the friction braking torque, the electromagnetic braking torque and the wheel braking torque is:

$$
\left\{\begin{array}{l}
T_{b c}=2 i_{c 3} \mu_{c} R_{p} \cdot F_{c} \\
T_{u}=T_{b e}+T_{b c}
\end{array}\right.
$$

In (5), $T_{u}$ refers to the total brake torque $\mathrm{N} \cdot \mathrm{m}$; $T_{b c}$ indicates the friction brake torque $\mathrm{N} \cdot \mathrm{m} ; R_{p}$ represents the brake pressure acting radius, $\mathrm{m} ; \mu_{c}$ suggests the friction coefficient of the brake wheel; $i_{c 3}$ is the transmission ratio from the brake disc to the wheels; other parameters are similar to those above.

According to (1), (2), (3) and (4), the total braking torque equation is obtained:

$$
T_{u}=\left\{\begin{array}{cc}
i_{c 2} \cdot T_{L} & \left(F_{N} \leq F_{s 0}\right) \\
i_{c 2} \cdot T_{L}+\frac{2 \eta_{1} i_{c 1} i_{c 3} \mu_{c} R_{p}}{R_{c 5} \cdot \tan \left(\alpha_{s}+\rho_{s}\right)} \cdot\left(T_{L}-T_{s}\right)-2 i_{c 3} \mu_{c} R_{p} \cdot F_{s 0} & \left(F_{N}>F_{s 0}\right)
\end{array}\right.
$$

Define the screw thrust coefficient for $k_{L}$ and the elastic coefficient for $K_{\mathrm{s}}$ :

$$
\left\{\begin{array}{l}
k_{L}=\frac{\eta_{1}}{R_{c 5} \cdot \tan \left(\alpha_{s}+\rho_{s}\right)} \\
k_{s}=2 \mu_{c} R_{p} \cdot \operatorname{sgn}\left(T_{L}-T_{s}\right) \cdot \operatorname{sgn}\left(F_{N}-F_{s}\right)
\end{array}\right.
$$

Then, the total brake torque equation can be expressed as:

$$
T_{u}=\left(i_{c 2}+i_{c 1} i_{c 3} k_{L} k_{s}\right) \cdot T_{L}-i_{c 1} i_{c 3} k_{L} k_{s} \cdot T_{s}-i_{c 3} k_{s} \cdot F_{s}
$$

In (7), the screw thrust $F_{N}=i_{c 1} k_{L}\left(T_{L}-T_{s}\right) \cdot \operatorname{sgn}\left(T_{L}-T_{s}\right)$.

By (3), (6) and (8), it is known that the total braking torque of the EMCB system is a continuous function of the motor braking torque $T_{L}$, and the motor braking torque can be freely distributed in a certain range [7]. As a result, the braking force distribution curve decided by EMCB regenerative braking system is freely controlled in a certain range. But the first order function $d T_{u} / d T_{L}$ is discontinuous. In order to make the total braking torque $T_{u}$ continuous and smooth, $d T_{u} / d x_{p}$ is continuous by designing the intermediate parameter $x_{p}$. The relationship between the total braking torque $T_{u}$ and the motor braking torque $T_{L}$ and $x_{p}$ can be expressed:

$$
\left\{\begin{array}{l}
T_{L}=\frac{a_{p} b_{p}^{2}+b_{p} x_{p}+i_{c 1} i_{c 3} k_{L} k_{s} \cdot T_{s}+i_{c 3} k_{s} \cdot F_{s}}{i_{c 2}+i_{c 1} i_{c 3} k_{L} k_{s}} \\
T_{u}=a_{p} x_{p}^{2}+b_{p} x_{p}
\end{array}\right.
$$

In (9), ${ }^{a_{p}}$ and ${ }^{b_{p}}$ are braking gain factors of $\mathrm{N} \cdot \mathrm{m}$ respectively; ${ }^{x_{p}}$ refers to non-dimensional intermediate parameter, ranged in $0 \sim 100$ in this paper.

\section{Results}

According to the coupled mechanism of former EMCB system, in this paper, small electric vehicles with four-wheel electromagnetic mechanical coupled regenerative braking system, are shown in Table 1. 
The slip rate of four wheel is independently controlled. Construct ABS control strategy in Matlab/Simulink, build the whole vehicle dynamics model in CarSim, and establish Matlab/Simulink and CarSim combination simulation platform. Taking the vehicle straight bend braking condition on the docking road and curve braking condition on low adhesion force road as an example, we make a simulation analysis of the wheel slip rate, the braking energy recovery, the braking stability, the braking efficiency and so on in the braking process.

\begin{tabular}{|c|c|}
\hline Parameters & Value \\
\hline Weight with full equipment $\mathrm{m} /(\mathrm{kg})$ & 1231 \\
\hline Distance from the front wheel to centeriod $a /(\mathrm{m})$ & 1.265 \\
\hline Distance from the latter wheel to centeriod $b /(\mathrm{m})$ & 1.335 \\
\hline Braking initial velocity $v_{x} /(\mathrm{km} / \mathrm{h})$ & 60 \\
\hline Motor peak power $\mathrm{P} /(\mathrm{kW})$ & 16 \\
\hline Peak torque of motor $\mathrm{T}(\mathrm{N} \cdot \mathrm{m})$ & 120 \\
\hline Motor rated power $\mathrm{P}_{\mathrm{e}} /(\mathrm{kW})$ & 11 \\
\hline Motor rated speed $\mathrm{n} /(\mathrm{r} / \mathrm{min})$ & 1200 \\
\hline
\end{tabular}

\subsection{Vehicles straight line braking on the docking road}

The high attached road is 0.6 , the low attached road is 2, and the type of the road is the high attached road $\rightarrow$ the low attached road $\rightarrow$ the high attached road. The slip rate of four wheels remained at around $20 \%$, and there was a slight fluctuation when the road is suddenly changed, but quickly returned to the steady state value. Using the usual algorithm in the braking end, the speed is lower than $2 \mathrm{~km}$, then exit the ABS control, the slip rate quickly rising to $100 \%$. In addition to the fluctuation in the sudden change of the road, the control input is stable in change, which realizes the continuous state control, avoids the chattering of the system, and reduces the requirement of the implementation system. The braking force does not fluctuate violently with the change of the road surface, with very good braking responsibility.

Vehicles straight line braking performance on the docking road is shown in Figure 3. Side-slip angle, lateral displacement and lateral acceleration are close to zero, with good braking stability; longitudinal acceleration maintains near the peak both in the high attached road and low attached road, which is rapidly smooth with the road characteristics change and has very good braking efficiency and braking responsiveness.

As shown in Figure 3, the change trend of vertical speed and braking strength are basically the same, and the average braking strength is $0.5 \mathrm{~g}$; braking energy recovery rate, with reversely steady change of the braking intensity, fluctuates in the vicinity of $25 \%$ and at last reaches $23.3 \%$. It effectively improves the braking energy recovery rate on the electric vehicle ABS braking conditions and thereby increases the mileage of the electric vehicles.

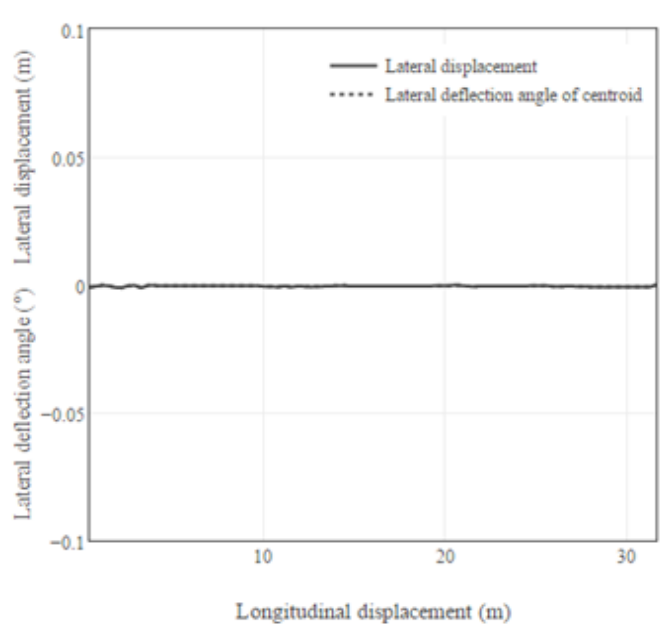

(a) The relationship between longitudinal displacement and the lateral displacement and lateral deflection angle of centroid

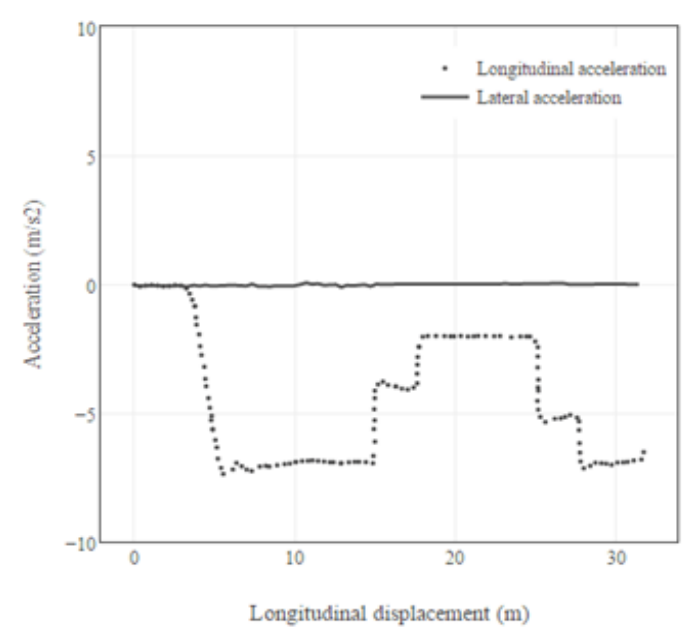

(b) The relationship between longitudinal displacement and acceleration 


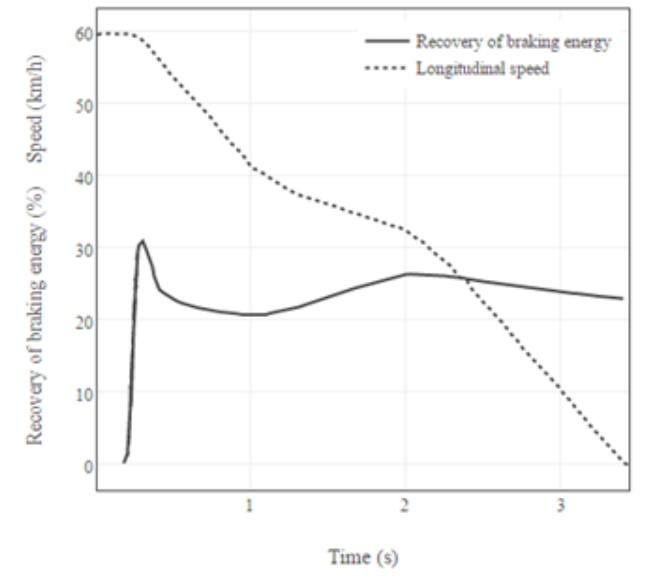

(c) The relationship between time and braking energy recovery rate and vehicle speed

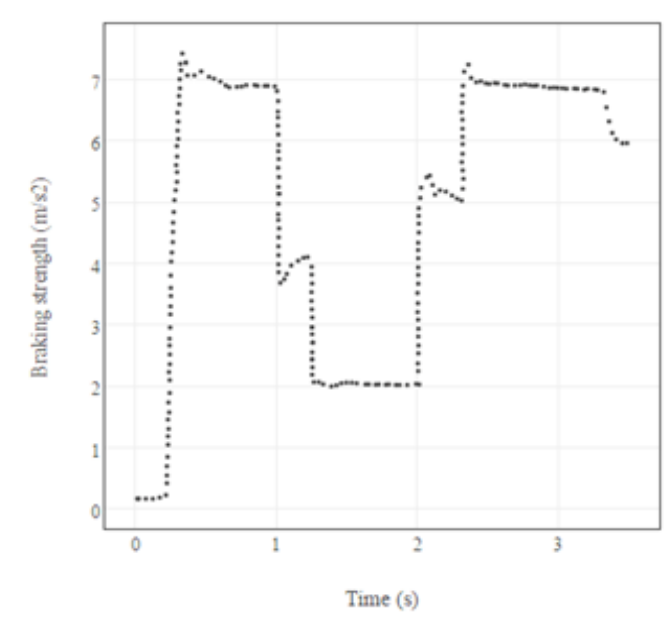

(d) The relationship between time and braking acceleration

Figure 3: $(a, b, c, d)$ Braking performance of vehicles on the docking road surface

\subsection{Vehicle cornering braking under low attached road}

The road surface adhesion coefficient of the cornering braking process under the low attached road surface is set to 0.4 , the braking start time is $0.2 \mathrm{~s}$, and the steering wheel angle steps from $(0.5 \mathrm{~s}$, $0 \mathrm{deg}$ ) to $(0.6 \mathrm{~s}, 180 \mathrm{deg})$. As shown in Figure 4 , sideslip angle change range is $-13 \mathrm{deg} \sim 8.6 \mathrm{deg}$, and the maximum lateral acceleration is $0.28 \mathrm{~g}$. When the tire force reaches the limit of road adhesion force, with the increase of lateral acceleration, the longitudinal acceleration decreases. The wheel load change caused by the longitudinal acceleration and the lateral acceleration makes the braking force under the equivalent slip rate shows a concave parabola.

The control input plays a role in the ABS control strategy and turns to the initial stage of rapid vibration to adjust the braking force, so the slip rate converges quickly to meet the target value.
And then the change is relatively stable, which avoids the high frequency vibration of the discrete control, achieves a continuous state control, and improves the system control performance.

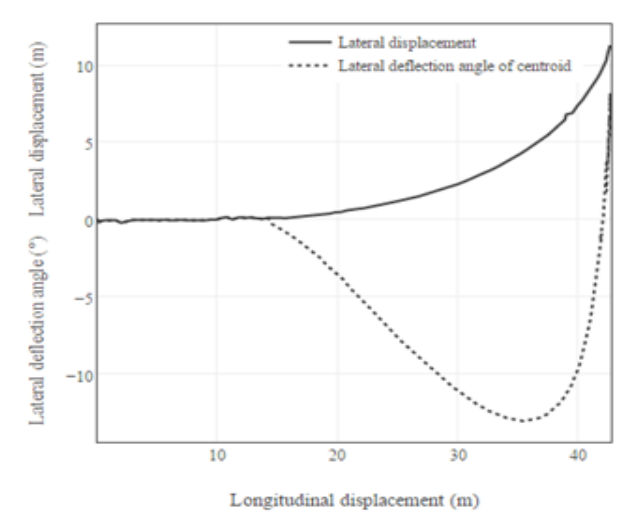

(a) The relationship between longitudinal displacement and the lateral displacement and lateral deflection angle of centroid

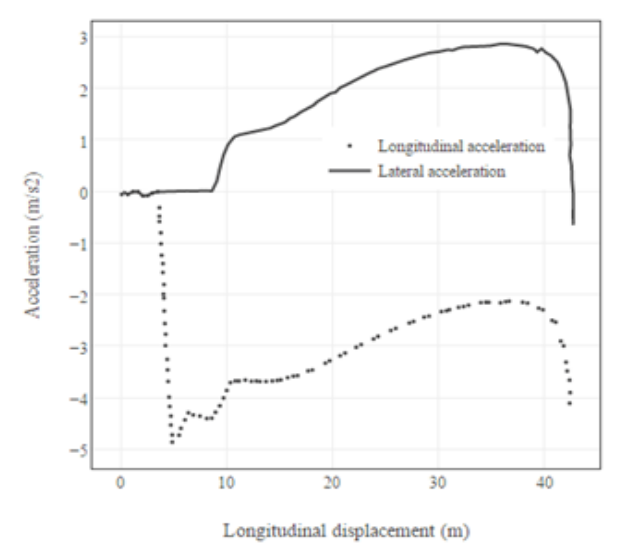

(b) The relationship between longitudinal displacement and acceleration

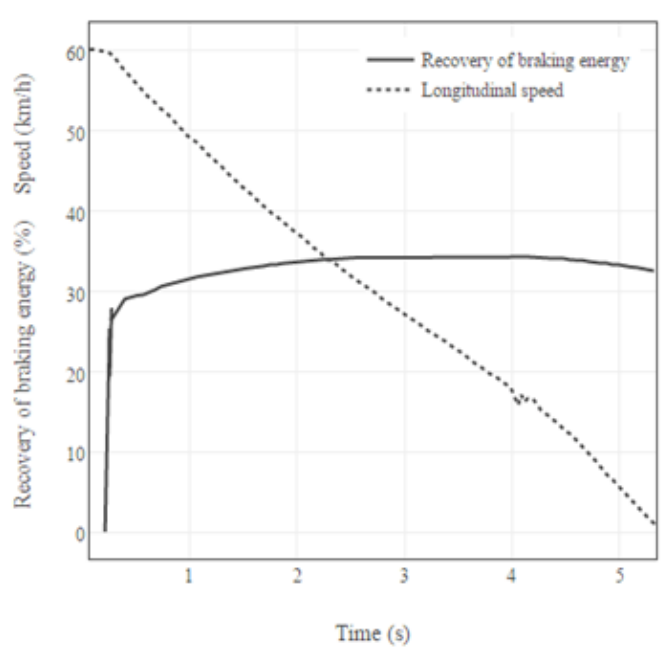

(c) The relationship between time and braking energy recovery rate and vehicle speed 


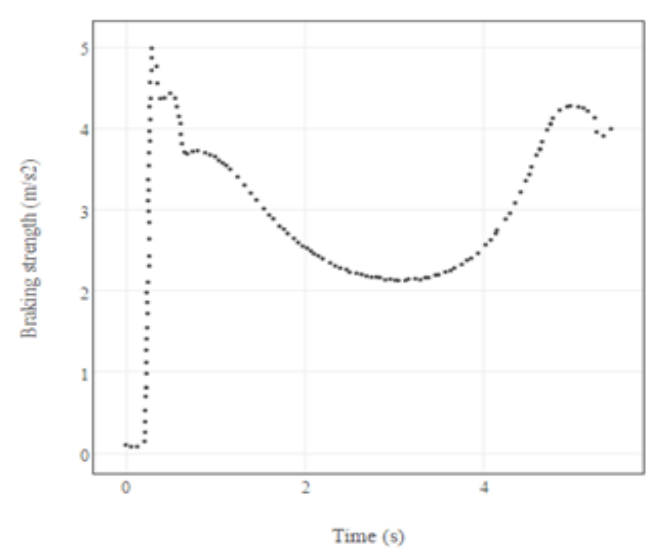

(d) The relationship between time and braking acceleration

Figure 4: $(a, b, c, d)$ Curve braking performance of vehicles on low adhesion force road

New type EMCB electromagnetic mechanical coupled regenerative braking system integrates the advantages of regenerative braking and electronic mechanical brake, and realizes the friction brake in the case of not consuming additional friction braking kinetic energy, which simplifies the system structure, reduces the control parameters, and improves the control performance of the system. Research results show that:

(1) In the straight line braking on the docking road, slip control is good and has excellent braking stability and braking efficiency; ABS continuous state control strategy based on EMCB has good response and robustness, which avoids the high frequency buffeting caused by excessive response of road surface interference.

(2) In the cornering brake on the low attached road, the sliding rate control is stable, and the ABS continuous state control strategy ensures the stability and braking efficiency of the large angle step steering curve brake under the condition of the maximum adhesion road surface.

(3) ABS continuous state control based on the electromagnetic mechanical coupled regenerative braking system improves the shortcomings of the traditional ABS discrete switching control, and avoids the high frequency chattering of the system, which has a better slip rate control performance, improves brake energy recovery rate and braking comfort, and increases the mileage of electric vehicles.

\section{Conclusion}

Based on the technical status and research results of regenerative braking and distribution driving of electric vehicles, this paper presents a new type of electromagnetic mechanical coupled regenerative braking system (EMCB).
It integrates the advantages of electronic mechanical braking, wheel motor regenerative braking, and direct distribution drive, makes use of the integrated braking / driving control system, and realizes the electromagnetic mechanical coupled regenerative braking in the case of not consuming additional friction brake kinetic energy, which provides a new means for electric vehicle stability control and unmanned technology. This paper mainly draws the following conclusions through the research: ABS continuous state control strategy of the new type electromagnetic mechanical coupled regenerative braking system has good response and robustness, and avoids high frequency chattering caused by the excessive response to the road surface disturbance in the synovial control area. In addition, it ensures the stability and braking efficiency of the large angle step steering curve brake under the condition of the maximum adhesion road surface, has a better slip rate control performance, and improves the brake energy recovery rate and braking comfort.

\section{References}

[1] Hu, X., Murgovski, N., Johannesson, L. M., \& Egardt, B. (2014). Comparison of three electrochemical energy buffers applied to a hybrid bus powertrain with simultaneous optimal sizing and energy management. Intelligent Transportation Systems IEEE Transactions on,15(3), 1193-1205.

[2] Sovran, G., \& Blaser, D. (2006). Quantifying the Potential Impacts of Regenerative Braking on a Vehicle's Tractive-Fuel Consumption for the U.S. European, and Japanese Driving Schedules. Congrès Français de Thermique.

[3] Zhang, R., \& Yao, E. (2015). Eco-driving at signalised intersections for electric vehicles. Intelligent Transport Systems Iet, 9(5), 488-497.

[4] Hakiai, M., Taichi, T., Shoda, M., Koizumi, T., Ashikaga, T., \& Shimizu, H. (1997). Brake system of "Eco-Vehicle". EVS14.

[5] Clarke, P., Muneer, T., \& Cullinane, K. (2010). Cutting vehicle emissions with regenerative braking. Transportation research part D: transport and environment, 15(3), 160-167.

[6] González-Gil, A., Palacin, R., Batty, P., \& Powell, J. P. (2014). A systems approach to reduce urban rail energy consumption. Energy Conversion and Management, 80, 509-524.

[7] Alipour, H., Sabahi, M., \& Sharifian, M. B. B. (2015). Lateral stabilization of a four-wheel independent drive electric vehicle on slippery roads. Mechatronics, 30, 275-285. 